M. Matsuda

Nagoya Math. J.

Vol. 68 (1977), 17-19

\title{
AN APPLICATION OF RITT'S LOW POWER THEOREM
}

\author{
MICHIHIKO MATSUDA
}

\begin{abstract}
Consider an algebraic differential equation $F=0$ of the first order. A rigorous definition will be given to the classical concept of "particular solutions" of $F=0$. By Ritt's low power theorem we shall prove that a singular solution of $F=0$ belongs to the general solution of $F$ if and only if it is a particular solution of $F=0$.
\end{abstract}

\section{§ 0. Introduction}

Let $k\{y\}$ be the differential polynomial algebra in a single indeterminate $y$ over an algebraically closed differential field $k$ of characteristic zero, and $F$ be an algebraically irreducible element of $k\{y\}$ of the first order. The totality $\Pi$ of those elements $A$ of $k\{y\}$ such that the remainder of $A$ with respect to $F$ is zero is an essential prime divisor of the perfect ideal $\{F\}$ in $k\{y\}$ generated by $F$. Let $I I, \Sigma_{1}, \cdots, \Sigma_{s}$ be the essential prime divisors of $\{F\}$. Then, each of the $\Sigma_{i}$ contains the separant $S$ of $F$ (Cf. [5, pp. 30-32]). Take and fix a universal extension $\Omega$ of $k$, the existence of which was proved by Kolchin [3, p. 771]. The manifold of $\Pi$ in $\Omega$ is called the general solution of $F$. A zero of $F$ in $\Omega$ is called a singular solution of $F=0$ if it is a zero of $S$. The manifold of $\Sigma_{i}$ in $\Omega$ consists of a single point for each $i$ (Cf. [5, p. 63]). A singular solution of $F=0$ is an element of $k$, because it is either a zero of the discriminant of $F$ with respect to $y^{\prime}$ or a zero of the initial of $F$.

Take a generic point $w$ of the general solution of $F$. Then, $w$ is transcendental over $k$. Hence, $k\left(w, w^{\prime}\right)$ is a one-dimensional algebraic function field over $k$, which will be denoted by $K$. We shall give a rigorous definition to the classical concept of "particular solutions" of $F=0$ as follows (Cf. [1, p. 257]):

DEFINITION. A singular solution $\eta$ of $F=0$ will be called a particReceived September 6, 1976. 
ular solution of $F=0$ if there exists a prime divisor $P$ of $K$ such that

$$
\nu_{P}\left(w^{\prime}-\eta^{\prime}\right) \geqq \nu_{P}(w-\eta)>0,
$$

where $\nu_{P}$ is the normalized valuation belonging to $P$.

This definition is independent of the choice of a generic point $w$ of the general solution of $F$.

By Ritt's low power theorem we shall prove the following:

THEOREM. A singular solution $\eta$ of $F=0$ belongs to the general solution of $F$ if and only if $\eta$ is a particular solution of $F=0$.

\section{§1. Proof of Theorem}

Suppose that $\eta$ is a singular solution of $F=0$. Then, $\eta$ is an element of $k$. Let $G$ denote the polynomial in $u, v$ obtained from $F$ by the replacement of $y=u+\eta, y^{\prime}=v+\eta^{\prime}$. Suppose that

$$
G=a_{0}(u) v^{n}+a_{1}(u) v^{n-1}+\cdots+a_{n}(u),
$$

where the $a_{i}$ are elements of $k[u]$. Unless $a_{i}=0$, we define $s_{i}$ as the least exponent of $u$ in $a_{i}$. If $a_{i}=0$, we do not define $s_{i}$. For $i=n, s_{n}$ can be defined, and $s_{n}>0$. The following lemma is a corollary of Ritt's low power theorem (Cf. [5, p. 65]):

LEMMA. The singular solution $\eta$ belongs to the general solution of $F$ if and only if we have the inequality

$$
s_{n} \geqq s_{i}+n-i
$$

for some $i$ different from $n(0 \leqq i<n)$.

Let us make Puiseux diagram in $G$. Then, we have rational numbers $\mu_{1}, \cdots, \mu_{m}$ and subscripts $i_{0}, i_{1}, \cdots, i_{m}$ of the $a$ such that they satisfy the following four conditions:

(i) $0 \leqq i_{0}<i_{1}<\cdots<i_{m}=n$;

(ii) $0<\mu_{1}<\ldots<\mu_{m}$;

(iii) for each $j(1 \leqq j \leqq m)$,

$$
s_{p}+\mu_{j}(n-p)=s_{q}+\mu_{j}(n-q), \quad p=i_{j-1}, \quad q=i_{j} ;
$$

(iv) $s_{i}+\mu_{j}(n-i) \geqq \tau_{j}$

for all $i, j(0 \leqq i \leqq n, 1 \leqq j \leqq m)$, where $\tau_{j}$ is the number given by the equality (3). 
Let $P$ be a prime divisor of $K$ such that

$$
\nu_{P}(w-\eta)>0, \quad \nu_{P}\left(w^{\prime}-\eta^{\prime}\right)>0 \text {. }
$$

Then, we have

$$
\nu_{P}\left(w^{\prime}-\eta^{\prime}\right)=\mu_{h} \nu_{P}(w-\eta)
$$

for some $h$. Conversely, for each $h(1 \leqq h \leqq m)$, there exists some prime divisor $P$ of $K$ which satisfies (4) and (5) (Cf. [2, Chap. 2], [4, Chap. 13]).

Because of (ii), there exists a prime divisor $P$ of $K$ satisfying (1) if and only if $\mu_{m} \geqq 1$. The inequality (2) holds for some $i$ different from $n(0 \leqq i<n)$ if and only if $\mu_{m} \geqq 1$. Hence, we have our Theorem by Definition and Lemma.

\section{§2. An example}

Let $k_{0}$ be an algebraically closed field of characteristic zero, and $k_{0}(x)$ be the one-dimensional rational function field over $k_{0}$. We set $x^{\prime}=1$, and $a^{\prime}=0$ for all elements $a$ of $k_{0}$. Suppose that $k$ is the algebraic closure of $k_{0}(x)$, and that

$$
F=x^{2}\left(y^{\prime}\right)^{2}+(2 x+y) y y^{\prime}+y^{2} .
$$

Then, the singular solutions of $F=0$ are 0 and $-4 x$. The former is a particular solution of $F=0$, and the latter is not.

Let $t$ denote $x+w / w^{\prime}$. Then, $t$ is a constant. We have $w=t^{2}(x-t)^{-1}$ and $w^{\prime}=-t^{2}(x-t)^{-2}$. Hence, $k\left(w, w^{\prime}\right)=k(t)$ with $t^{\prime}=0$.

\section{BIBLIOGRAPHY}

[1] A. R. Forsyth, Theory of differential equations, Part II, Ordinary equations, not linear, Vol. II, Cambridge Univ. Press, London, 1906.

[2] K. Iwasawa, Theory of algebraic function fields (in Japanese), Iwanami Shoten, Tokyo, 1952.

[ 3 ] E. R. Kolchin, Galois theory of differential fields, Amer. J. Math., 75 (1953), 753-824.

[4] É. Picard, Traité d'analyse, Tome II, $2^{\circ}$ Édition, Gauthier-Villars, Paris, 1905.

[ 5 ] J. F. Ritt, Differential algebra, Amer. Math. Soc. Colloq. Publ. Vol. 33, New York, 1950.

Department of Mathematics

Osaka University 